\title{
Facile Construction of Functionalized GO Nanocomposites with Enhanced Antibacterial Activity
}

\author{
Lei Jiang ${ }^{1,2}$, Zhongjie Zhu ${ }^{2}$, Yanyi Wen ${ }^{2}$, Shan Ye ${ }^{2}$, Chen Su ${ }^{2}$, Rui Zhang ${ }^{2, *}$ and Wei Shao ${ }^{1,2, *}$ \\ 1 Jiangsu Co-Innovation Center of Efficient Processing and Utilization of Forest Resources, \\ Nanjing Forestry University, Nanjing 210037, China \\ 2 College of Chemical Engineering, Nanjing Forestry University, Nanjing 210037, China \\ * Correspondence: zhangrui@njfu.edu.cn (R.Z.); w.shao@njfu.edu.cn (W.S.)
}

Received: 17 May 2019; Accepted: 5 June 2019; Published: 26 June 2019

\begin{abstract}
The development of antimicrobial materials with sustained drug release performance is of great importance. Graphene oxide (GO) is considered to be an ideal drug carrier. In this study, tetracycline hydrochloride (TC) was loaded onto polyethyleneimine-functionalized GO (PG) to fabricate TC/PG nanocomposites. The success of the fabrication was confirmed by zeta potential, TEM, FTIR, and Raman analyses. The TC/PG nanocomposites showed a controlled and sustained drug release behavior, and a pseudo second order kinetic model was employed to illustrate the release mechanism. The antibacterial activity was studied using the disk diffusion method against Escherichia coli and Staphylococcus aureus. The TC/PG nanocomposites exhibited great bacterial inhibition performance. The results indicate that the fabricated TC/PG nanocomposites with effective antibacterial activity have great potential in antibacterial applications.
\end{abstract}

Keywords: graphene; polyethyleneimine; controlled release; antibacterial

\section{Introduction}

Graphene, a two-dimensional (2D) carbon material that is one atom thick, has attracted the attention of researchers in various fields due to its exceptional optical, electronic, thermal, and mechanical properties [1-4]. Graphene oxide (GO) is a derivative of graphene and owns many functional groups, including epoxide, hydroxyl, carboxyl, and carbonyl groups [5]. GO has great potential in biomedical applications due to its high surface area, good biocompatibility, antibacterial properties, and capacity for facile functionalization using both chemical and biological methods [6]. GO has attracted attention in drug-delivery and controlled-release applications due to its intrinsic conjugated $\pi$-structure and ease of functionalization [7]. Nanosized fluorinated GO sheets for a targeted drug delivery system with switchable fluorescence, high near-infrared absorption, and controlled drug release performance were constructed in [8]. Poly(amidoamine)-modified GO as a siRNA vector was developed and exhibited great potential in gene-based antitumor therapy due to its $\mathrm{pH}$-triggered release performance [9]. A chlorogenic acid-GO nanocomposite with sustained and $\mathrm{pH}$-dependent release performance was successfully synthesized and exhibited an insignificant toxicity effect towards a normal cell line and an enhanced toxic effect towards the evaluated cancer cell lines [10].

In recent years, the antibacterial performance of GO nanosheets has been widely studied, and several antibacterial mechanisms have been proposed, including nanoknives, trapping or wrapping, and oxidative stress [11-13]. The physicochemical properties of GO, including its shape, dimensions, and surface functionalization, can influence its antibacterial behavior [14]. GO with sharp edges can easily permeate into a bacterial membrane due to this unique structure [15]. GO with a larger 
lateral size has been reported to exhibit a stronger bacteria killing ability [16]. However, in another study, GO of a smaller size was reported to exhibit higher antibacterial activity than GO of a larger size [17]. The functionalization of GO enriches graphene-based nanomaterial applications, which range from electrical conductivity to antibacterial use [18]. GO/quaternary ammonium salt nanocomposites were successfully fabricated by non-covalent functionalization and showed controlled drug release and good antibacterial performance [19]. Poly[5,5-dimethyl-3-(3'-triethoxysilylpropyl)hydantoin]/GO nanocomposites were synthesized through covalent functionalization and exhibited great antibacterial activity [20]. A UiO-66/GO membrane was fabricated and shown to possess good antimicrobial activity that would effectively prevent biofouling [21]. Moreover, metal and metal-oxide nanoparticles were loaded onto GO nanosheets to endow them with ideal antibacterial performance [22-24]. Silver-doped laser-induced graphene was shown to exhibit potent surface antibacterial and anti-biofilm activity [25]. A novel copper nanoparticles-decorated graphene sponge was synthesized successfully and could be used as a novel bactericidal filter due to its high antibacterial activity [26]. GO nanosheets were applied as drug carriers for antibiotics in order to achieve outstanding synergistic antibacterial effects [27].

Polyethyleneimine (PEI) is considered to be an ideal surface modifier due to its intrinsic polycationic performance with a high charge density and a high isoelectric point $(>10)$ [1]. Therefore, it is believed that the fabrication of PEI-functionalized GO (PG) nanosheets will endow GO with a high positive surface charge and increased stability. These PG nanosheets with a positive charge could easily adsorb onto negatively charged bacterial cell membranes, giving the fabricated PG nanosheets enhanced antibacterial activity. The formation of electrostatic interactions, hydrogen bonds, and covalent bonds between PEI and GO provide PG nanosheets with a supramaximal specific surface area, which makes PG nanosheets an ideal drug carrier [28]. PEI-functionalized GO was able to deliver DNA effectively, suggesting that it is an ideal candidate for gene delivery $[29,30]$. Photothermal, silver-loaded, PEI-mediated magnetic GO composites were successfully prepared in [31]. In our previous study, PEI was successfully covalently linked to GO using EDC.HCl as the catalyst, and it exhibited a synergistic antibacterial effect [32].

In order to develop new antibacterial agents with highly reduced doses of antibiotics for combating micro-organisms and suppressing bacterial activity, tetracycline hydrochloride (TC), which has broad-spectrum antibacterial activity, was loaded onto PG to produce TC/PG nanocomposites. In this study, PEI was used to functionalize GO without a catalyst. The surface morphology and hydrophilicity were studied. The TC release behavior and antibacterial performance of the TC/PG nanocomposites were evaluated.

\section{Materials and Methods}

\subsection{Fabrication}

One gram $(1 \mathrm{~g})$ of PEI $\left(\mathrm{M}_{\mathrm{w}}=70,000,50 \%\right)$ was added to a $2 \mathrm{mg} / \mathrm{mL} 50 \mathrm{~mL}$ GO suspension (XFNANO Materials Tech Co. Ltd., Nanjing, China) and stirred for $24 \mathrm{~h}$. The suspension was centrifugated at $12,000 \times \mathrm{g} \mathrm{rpm}$ for $10 \mathrm{~min}$, rinsed with de-ionized water, and freeze-dried. A $20 \mathrm{~mL}$ mixture of PG and TC was prepared with a fixed concentration $(10 \% w / w)$. Then, the mixture was filtered through a cellulose acetate membrane with a $0.22 \mu \mathrm{m}$ pore size. Finally, TC/PG nanocomposites were obtained using the freeze-dry method.

\subsection{Characterization}

Transmission electron microscopy (TEM) pictures were obtained with a JEM-2100 TEM instrument at an accelerating voltage of $200 \mathrm{kV}$. Fourier-transform infrared (FTIR) analysis was carried out on a Spectrum Two FTIR Spectrometer (Perkin Elmer, Akron, OH, USA). Raman spectra were obtained on a DXR Smart Raman spectrometer (Thermo Fisher Scientific, Waltham, MA, USA) using a laser excitation of $532 \mathrm{~nm}$. 


\subsection{Release Behavior}

Five milligrams ( $5 \mathrm{mg}$ ) of nanocomposite was placed into a $3500 \mathrm{Da}$ dialysis bag, immersed in $50 \mathrm{~mL}$ of phosphate-buffered saline (PBS) buffer $(\mathrm{pH}=7.4)$, and sealed. Then, $2.5 \mathrm{~mL}$ of solution was taken out at fixed time intervals and tested at a wavelength of $356 \mathrm{~nm}$ using a SHIMADZU 2450 UV spectrophotometer (Shimadzu Corporation, Kyoto, Japan). Finally, $2.5 \mathrm{~mL}$ of fresh PBS buffer solution was added into the system, and the concentration of released TC was measured.

\subsection{Antibacterial Activity}

\subsubsection{Inhibition of Growth Effect}

One hundred microliters $(100 \mu \mathrm{L})$ of pre-cultured Escherichia coli and Staphylococcus aureus suspensions with a concentration of $1 \times 10^{6}$ colony-forming units (CFU)/mL were added into $40 \mathrm{~mL}$ of Tryptone Soya Broth (TSB) medium, respectively. Then, different amounts of TC/PG nanocomposites were added to achieve concentrations of $0.5,1,2,4$, and $8 \mu \mathrm{g} / \mathrm{mL}$. The mixtures were incubated at $37^{\circ} \mathrm{C}$ for $8 \mathrm{~h}$ at $150 \mathrm{rpm}$. Then, $2.5 \mathrm{~mL}$ of supernatant was taken out and tested at a wavelength of $600 \mathrm{~nm}$ (optical density (OD)) using a UV spectrophotometer (SHIMADZU 2450). The inhibition activity $(I, \%)$ was calculated as follows:

$$
I(\%)=\frac{O D_{\text {control }}-O D_{\text {sample }}}{O D_{\text {control }}} \times 100 \%
$$

where $O D_{\text {control }}$ is the OD value at $600 \mathrm{~nm}$ of the bacterial supernatant without any treatment as the control, and $O D_{\text {sample }}$ is the $O D$ value at $600 \mathrm{~nm}$ of the bacterial supernatant with treatment with different samples, respectively.

Furthermore, the supernatant was diluted to $10^{-3}$ times the concentration, dispersed onto TSB plates, and incubated at $37^{\circ} \mathrm{C}$ for $24 \mathrm{~h}$. The number of surviving micro-organisms was observed and pictures were taken.

\subsubsection{Fluorescent Stain}

One milligram ( $1 \mathrm{mg}$ ) of nanocomposite was added to $20 \mathrm{~mL}$ E. coli and S. aureus suspensions containing $1 \times 10^{6} \mathrm{CFU} / \mathrm{mL}$ cells, respectively. The suspensions were incubated at $120 \mathrm{rpm}$ at $37^{\circ} \mathrm{C}$ for $2 \mathrm{~h}$, followed by staining with propidium iodide (PI) and SYTO9 for 15 min under dark conditions, respectively. Then, they were imaged by an Olympus Fluorescence Microscope (IX53, Olympus, Tokyo, Japan). The bacterial suspension without treatment was used as the control.

\subsubsection{Inhibition Zone Behavior}

A TC/PG nanocomposite-loaded paper was cut into round shapes with a diameter of $10 \mathrm{~mm}$, and an ultraviolet lamp was applied for $60 \mathrm{~min}$ to sterilize them. Lawns of E. coli and S. aureus with about $1 \times 10^{5} \mathrm{CFU} /$ plate on the Tryptone Soya Agar (TSA) were pre-prepared. The sterilized specimens were placed upon them carefully. Then, the plates were incubated at $37^{\circ} \mathrm{C}$ for one day. The bacterial inhibition ability was estimated by measuring the inhibition zone diameter.

\subsubsection{Bacterial Morphologies}

Twenty microliters $(20 \mu \mathrm{L})$ of bacterial suspension was added to $40 \mathrm{~mL}$ of TSB and incubated at $120 \mathrm{rpm}$ at $37^{\circ} \mathrm{C}$ for $4 \mathrm{~h}$. Then, $1 \mathrm{mg}$ of TC/PG nanocomposite was added. The bacteria with and without nanocomposite treatment were fixed using $4 \%$ glutaraldehyde for $24 \mathrm{~h}$. The specimens were dehydrated with sequential ethanol treatments for $10 \mathrm{~min}$ and lyophilized. They were fixed on an aluminum stub, coated with platinum, and observed by a JSM-7600F SEM (JEOL, Tokyo, Japan). 


\subsubsection{Protein Leakage}

The pre-incubated E. coli and S. aureus suspensions were centrifugated at $6000 \mathrm{rpm}$ for $5 \mathrm{~min}$, rinsed three times with $0.9 \% \mathrm{NaCl}$, and diluted in $0.9 \% \mathrm{NaCl}$ to obtain $\mathrm{OD}_{600}$ values of 1.5. Two hundred microliters $(200 \mu \mathrm{L})$ of TC/PG nanocomposite $(1 \mathrm{mg} / \mathrm{mL})$ was put in $20 \mathrm{~mL}$ of bacterial suspension. The control group without any sample was used. The above mixture was incubated at $120 \mathrm{rpm}$ at $37^{\circ} \mathrm{C}$ for $4 \mathrm{~h}$. Forty microliters $(40 \mu \mathrm{L})$ of supernatant was put into $400 \mu \mathrm{L}$ of working solution, which was obtained using an Enhanced BCA Protein Assay Kit (Beyotime, Shanghai, China). After incubation at $37^{\circ} \mathrm{C}$ for $30 \mathrm{~min}$, the absorption of the above solution was tested at a wavelength of $562 \mathrm{~nm}$. The protein leakage concentrations were calculated.

\subsubsection{Detection of Reactive Oxygen Species (ROS) Production}

Oxidative stress measurements were carried out with 2,3-bis(2-methoxy-4-nitro-5-sulfophenyl)-2Htetrazolium-5-carboxanilide (XTT, Invitrogen, Eugene, OR, USA) and Ellman's assays. XTT was added into a $0.01 \mathrm{M}$ PBS buffer solution to obtain a concentration of $0.4 \mathrm{mM}$. One hundred microliters $(100 \mu \mathrm{L})$ of a $2 \mathrm{mg} / \mathrm{mL}$ TC/PG nanocomposite dispersion was put in $2 \mathrm{~mL}$ of XTT solution and incubated for $2-6 \mathrm{~h}$ in the dark at $150 \mathrm{rpm}$. The mixture was filtered $(0.22 \mu \mathrm{m})$, and the supernatant was tested at a wavelength of $470 \mathrm{~nm}$ using a Spectrum Two UV Spectrometer. XTT solution without any sample was used as the control. Reduced glutathione (GSH) oxidation was measured using an Ellman's assay [33]. Briefly, GSH was added into $50 \mathrm{mM}$ bicarbonate buffer ( $\mathrm{pH}$ 8.6) to obtain a concentration of $0.8 \mathrm{mM}$. One hundred microliters $(100 \mu \mathrm{L})$ of TC/PG nanocomposite dispersion $(2 \mathrm{mg} / \mathrm{mL})$ was put in $2 \mathrm{~mL}$ of GSH bicarbonate buffer. It was incubated for 2-6 h in the dark at $150 \mathrm{rpm}$. Then, $1.5 \mathrm{~mL}$ of $50 \mathrm{mM}$ Tris- $\mathrm{HCl}$ and $30 \mu \mathrm{L}$ of $100 \mathrm{mM}$ DNTB (Molecular Probe) solution was put in the above mixture, and a product exhibiting a yellow color was formed. It was filtered $(0.22 \mu \mathrm{m})$, and the supernatant was tested at a wavelength of $412 \mathrm{~nm}$ using a Spectrum Two UV Spectrometer. GSH solution without any sample was applied as the negative control, and $1 \mathrm{mM} \mathrm{H}_{2} \mathrm{O}_{2}$ was put in $0.4 \mathrm{mM} \mathrm{GSH}$ solution as the positive control. The GSH loss percentage $\left(\mathrm{L}_{\mathrm{G}}\right)$ was determined using the following equation:

$$
L_{G}=\frac{A_{n}-A_{s}}{A_{n}} \times 100 \%
$$

where $A_{n}$ and $A_{s}$ are the absorption values of the negative control and samples, respectively.

\subsection{Statistical Analysis}

The OriginPro 8 software was used to analyze the data, which are expressed as mean \pm standard error (SE). Statistical differences were evaluated using a Student's $t$-test. A $p$-value of $<0.05$ was considered to be statistically significant.

\section{Results and Discussion}

\subsection{Morphology}

TEM images of GO and TC/PG nanocomposites are displayed in Figure 1. As shown in Figure 1A, the GO nanosheet exhibits an almost transparent single layer, which is consistent with the previous report [34]. Figure 1B shows the morphology of the TC/PG nanocomposite. Its transparency is clearly reduced after PEI functionalization. Figure $1 C$ is an enlarged image of the square location on the TC/PG nanocomposite shown in Figure 1B. It can be seen that the TC has spherical and dark spots, and is dispersed on the PG nanosheets uniformly, indicating that TC was successfully loaded onto the functionalized GO nanosheets. The Zeta potentials of the GO nanosheets, the PG nanosheets, and the TC/PG nanocomposites were tested. The GO nanosheets carry a negative charge, and the measured Zeta potential of the $2 \mathrm{mg} / \mathrm{mL}$ GO nanosheets is $-65.5 \pm 1.2 \mathrm{mV}$. The high negative charges can make it difficult for the GO nanosheets to approach the bacterial cell membrane due to the existence 
of a repulsive interaction. Since the PEI has positively charged mass amidogens, both the $2 \mathrm{mg} / \mathrm{mL}$ PG nanosheets and the $2 \mathrm{mg} / \mathrm{mL}$ TC/PG nanocomposites became positively charged, and their Zeta potentials were found to be $35.8 \pm 0.7 \mathrm{mV}$ and $20.3 \pm 0.3 \mathrm{mV}$, respectively. These positive charges help the TC/PG nanocomposites to approach the bacterial cell membrane.
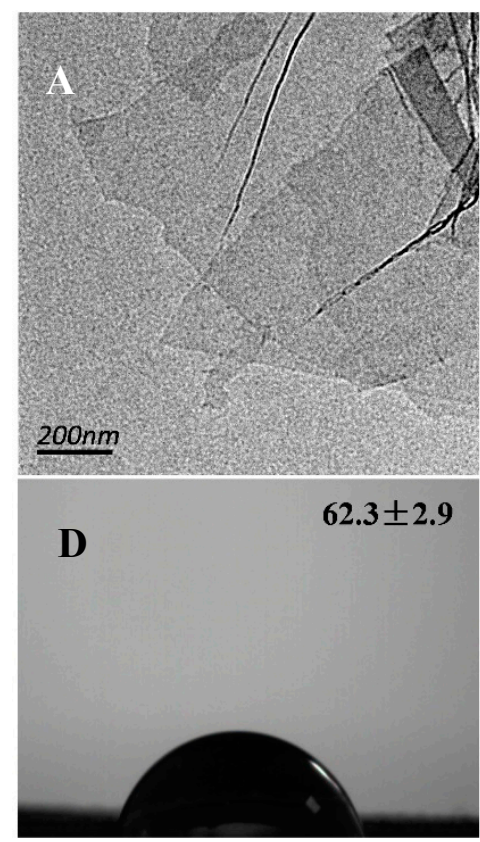

Figure 1. TEM images of graphene oxide (GO) (A) and tetracycline hydrochloride (TC)/polyethyleneimine (PEI)-functionalized GO (PG) (B,C). C shows a higher magnification of the square location in image B. Contact angle pictures of GO (D), PG (E), and TC/PG (F).

\subsection{FTIR and Raman Analysis}

FTIR spectra of GO nanosheets, PG nanosheets, and TC/PG nanocomposites are displayed in Figure 2A. The characteristic bands at 1720 and $1399 \mathrm{~cm}^{-1}$ were assigned to the stretching vibrations of $\mathrm{C}=\mathrm{O}$ and the bonding of $\mathrm{C}-\mathrm{OH}$ of $\mathrm{GO}$ (curve a), respectively [1]. After PEI functionalization (curve b), the characteristic peak at $1720 \mathrm{~cm}^{-1}$ disappears. The peaks at 2950 and $2810 \mathrm{~cm}^{-1}$ were attributed to $-\mathrm{CH}_{2}$ - stretching vibrations of the PEI. The peak located at $1655 \mathrm{~cm}^{-1}$ originated from $\mathrm{C}=\mathrm{O}$ stretching vibrations of amide bonds [35,36]. Moreover, the peak located at $1573 \mathrm{~cm}^{-1}$ corresponded to the N-H bond of the primary amino acid of PEI [37]. Therefore, we can confirm that a covalent bond has formed between GO and PEI. Moreover, a hydrogen-bonding interaction between GO and PEI could also exist, since GO has a large number of oxygen-containing functional groups and PEI chains have many $-\mathrm{NH}_{2}$ groups. In the TC/PG nanocomposite spectrum (curve c), a new peak located at $1456 \mathrm{~cm}^{-1}$ can be seen that corresponds to the $\mathrm{C}=\mathrm{C}$ vibration of the aromatic ring of $\mathrm{TC}$ [38]. Thus, we can confirm that the TC was successfully loaded onto the PG nanosheets.

Figure 2B shows the UV-vis absorption spectra of the GO nanosheets, the PG nanosheets, and the TC/PG nanocomposites, respectively. The spectrum for GO nanosheets (curve a) displays a sharp peak at $230 \mathrm{~nm}$ that corresponds to the electronic $\pi-\pi^{*}$ transitions of aromatic $C-C$ bonds [39]. The spectrum for PG nanosheets (curve b) shows that the peak at $230 \mathrm{~nm}$ vanished and another peak appeared at $260 \mathrm{~nm}$, which was attributed to the reduction ability of PEI that led to the formation of PEI-reduced graphene oxide (rGO) nanocomposites [40]. In the spectrum for the TC/PG nanocomposites, a new peak appeared at $356 \mathrm{~nm}$ that corresponded to the characteristic peak of TC [41]. Thus, we can verify that TCH was successfully loaded onto PG nanosheets. 


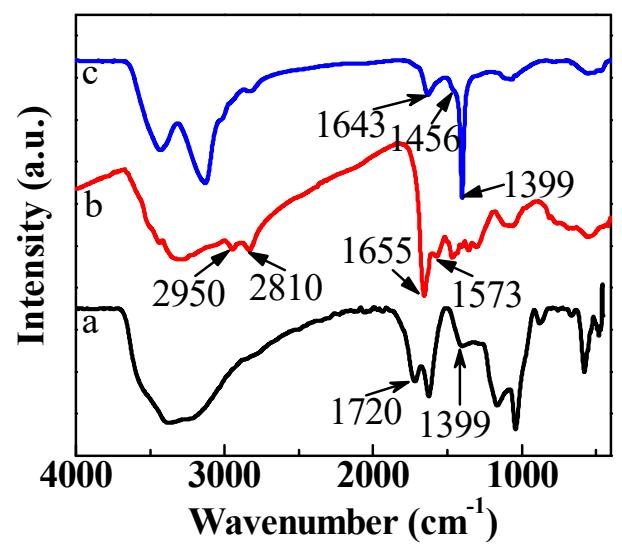

(A)

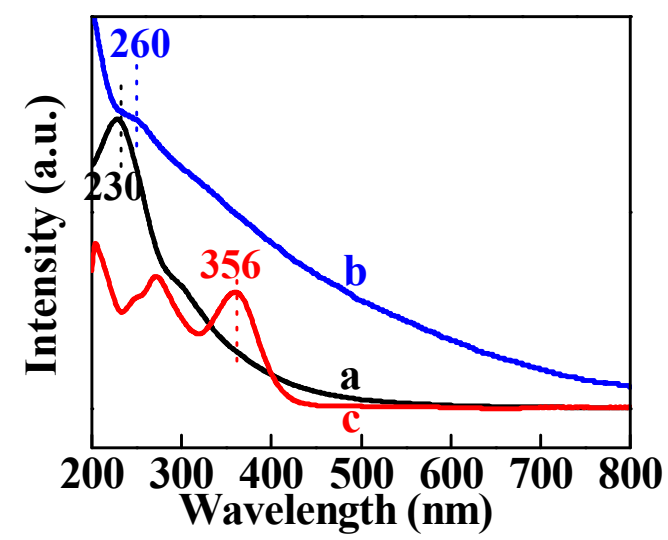

(B)

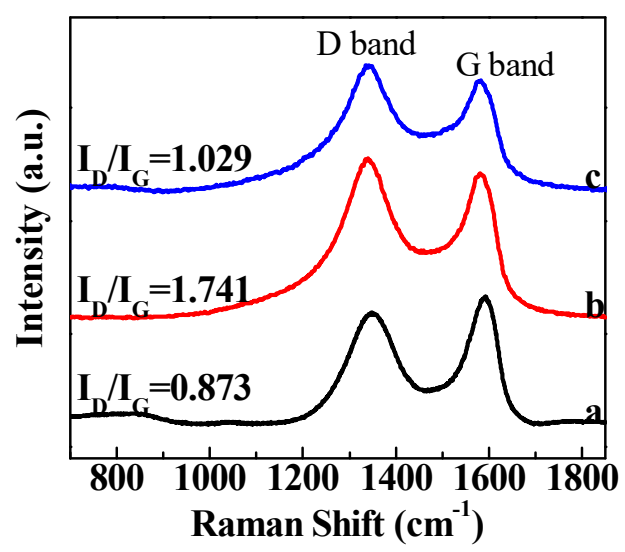

(C)

Figure 2. FTIR spectra (A), UV-vis absorption spectra (B), and Raman spectra (C) of GO nanosheets (a), PG nanosheets (b), and TC/PG (c) nanocomposites.

Raman spectroscopy was employed in order to illustrate the structural changes in functionalized GO. Raman spectra of GO nanosheets, PG nanosheets, and TC/PG nanocomposites are displayed in Figure $2 \mathrm{C}$. In the spectra for the GO nanosheets (curve a), the two bands located at $1580 \mathrm{~cm}^{-1}$ and $1352 \mathrm{~cm}^{-1}$ were denoted the $\mathrm{D}$ band and the $G$ band, respectively. The intensity ratio between the $\mathrm{D}$ band and the $G$ band $\left(\mathrm{I}_{\mathrm{D}} / \mathrm{I}_{\mathrm{G}}\right)$ was determined to assess the surface defect density. Although both $\mathrm{D}$ and $G$ bands exist in the Raman spectra of the PG nanosheets (curve b) and the TC/PG nanocomposites (curve c), their intensities have changed. The $\mathrm{I}_{\mathrm{D}} / \mathrm{I}_{\mathrm{G}}$ value of the GO nanosheets (curve a) was found to be 0.873 . The $\mathrm{I}_{\mathrm{D}} / \mathrm{I}_{\mathrm{G}}$ of the PG nanosheets (curve $\mathrm{b}$ ) increased to 1.741 , which may be due to the increased number of defects in the lattices and stretching based on the graphene layers that occurred, in part, because of the formation of chemical bonds between GO and PEI. In the case of the TC/PG nanocomposites (curve c), the $\mathrm{I}_{\mathrm{D}} / \mathrm{I}_{\mathrm{G}}$ was lower (1.029) than that of the PG nanosheets, because the TC that was loaded onto the nanocomposite covered some of the defects on the PG's surface.

\subsection{TC Release Performance}

The TC release profile is shown in Figure 3A. A rapid TC release behavior in the first $120 \mathrm{~min}$ was observed with about $52 \%$ of released TC, which is due to the 'burst-release' effect. Then, a controlled and sustained TC release behavior after $120 \mathrm{~min}$ was observed. The drug release mechanism was studied using mathematical modeling. A pseudo second order kinetic model was employed to illustrate the TC release mechanism. The correlation coefficient $\left(R^{2}\right)$ was used to identify the model that was suitable to describe the drug release mechanism. In this study, a pseudo second order kinetic model (Figure 3B) was considered to be a suitable model to illustrate the TC release behavior from 
the PG matrix, as the $\mathrm{R}^{2}$ was determined to be 0.97205 . This result is consistent with that reported by Barahuie et al., who found that the same drug release mechanism was exhibited using GO as the drug carrier [10].

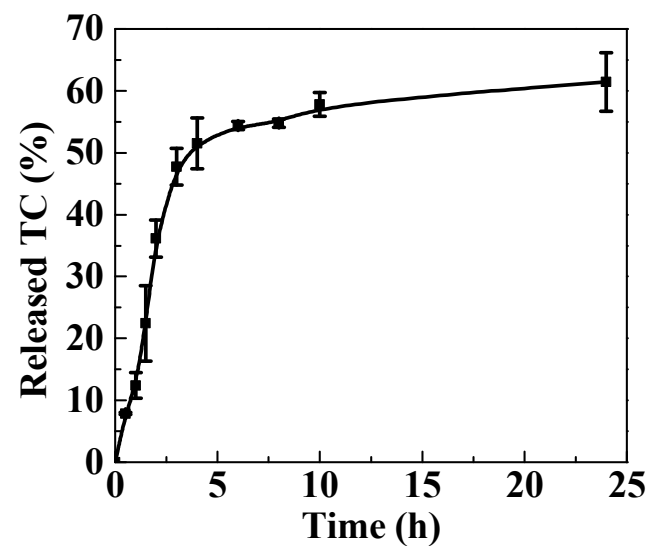

(A)

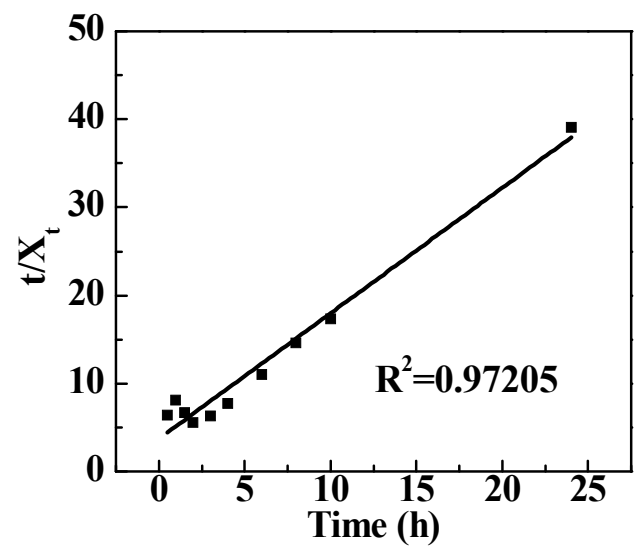

(B)

Figure 3. TC release behavior (A) and the release kinetic model (B) of the TC/PG nanocomposites.

\subsection{Antibacterial Performance}

Figure $4 \mathrm{~A}, \mathrm{C}$ show the numbers of bacterial colonies after treatment with different concentrations of TC/PG nanocomposites followed by dilution to $10^{-3}$ times the original concentration and culture on TSA plates for $24 \mathrm{~h}$. A large number of E. coli colonies was observed on the plate without TC/PG nanocomposite treatment (Figure 4A). The number of E. coli colonies decreased as the concentration of the TC/PG nanocomposite increased. No E. coli colonies were detected on the TSA plates after treatment with the $8 \mathrm{ug} / \mathrm{mL}$ TC/PG nanocomposite. The calculated inhibition activity is shown in Figure 4B. The inhibition activity of the $0.5 \mathrm{ug} / \mathrm{mL}$ TC/PG nanocomposite against $E$. coli was $28.5 \%$. The inhibition effect was enhanced as the concentration of the TC/PG nanocomposite increased. For the $8 \mathrm{ug} / \mathrm{mL}$ TC/PG nanocomposite, the inhibition activity reached $99.9 \%$. Therefore, the TC/PG nanocomposites have a highly concentration-dependent inhibition effect on E. coli. The TC/PG nanocomposites showed similar inhibition behaviour against $S$. aureus. TC/PG nanocomposites have a highly concentration-dependent inhibition effect on S. aureus. In the case of the $8 \mathrm{ug} / \mathrm{mL}$ TC/PG nanocomposite, no $S$. aureus colonies were found on the plate (Figure 4C), and the inhibition activity reached 99.5\% (Figure 4D). The TC/PG nanocomposites exhibit superior antibacterial activity against $E$. coli and S. aureus.

The antibacterial mechanism of TC/PG nanocomposites was studied by staining the treated bacteria using a LIVE/DEAD BacLight Bacterial Viability Kit (L13152). Strains, regardless of whether they are live with intact membranes or dead with damaged membranes, can be stained by SYTO 9 , and exhibit fluorescent green. Dead strains can be stained by PI due to their damaged membranes, which emit red fluorescence. So, the number of dead or alive bacteria is represented by red or green fluorescent dots, respectively. Pictures of the stained cells are shown in Figure 5. For the control group, little red fluorescence is displayed, indicating that the untreated cells are alive. Accordingly, after treatment with TC/PG nanocomposites, almost all of tested cells exhibited fluorescent green. This result indicates that the cell membranes were damaged after being exposed to the TC/PG nanocomposites. In other words, E. coli and S. aureus cell death was induced via destruction of the integrity of the cell membrane by the TC/PG nanocomposites. This confirms the excellent antibacterial activity of the TC/PG nanocomposites. 


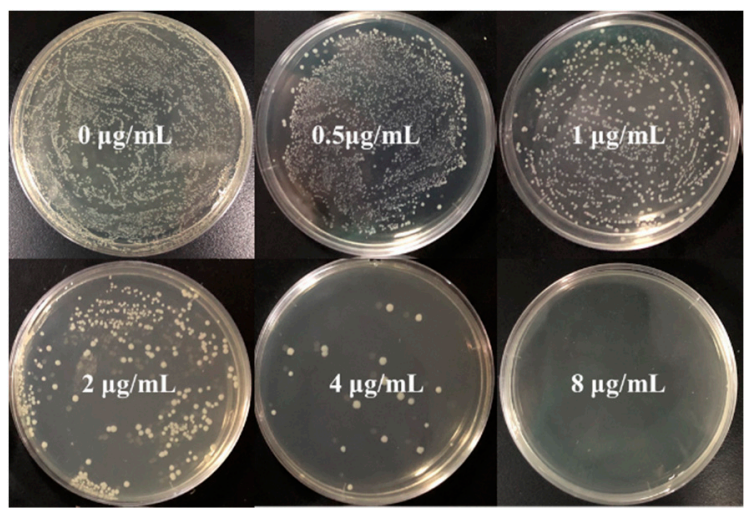

(A)

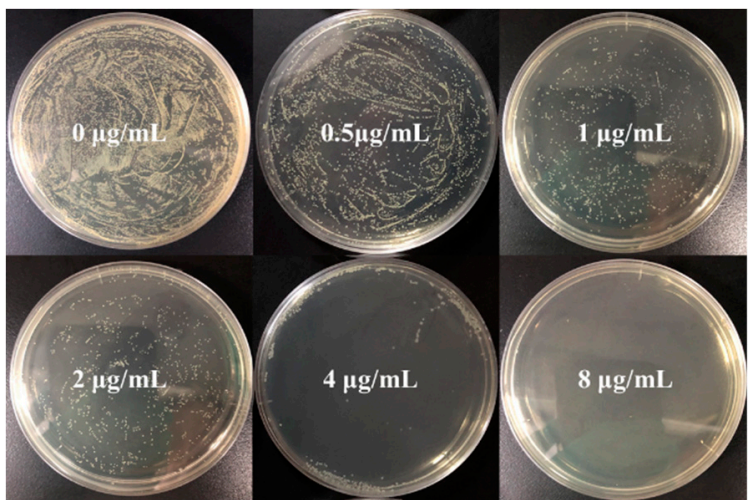

(C)

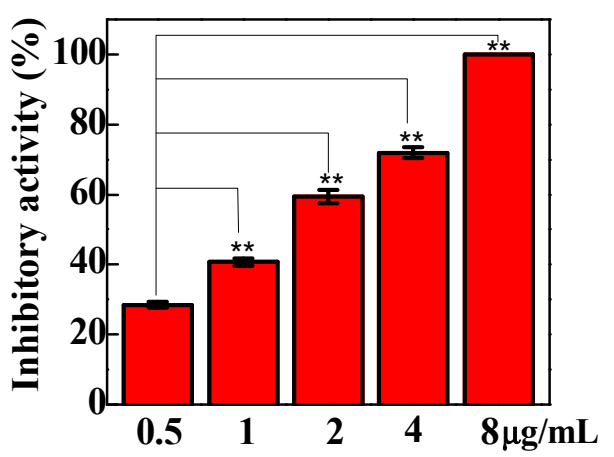

(B)

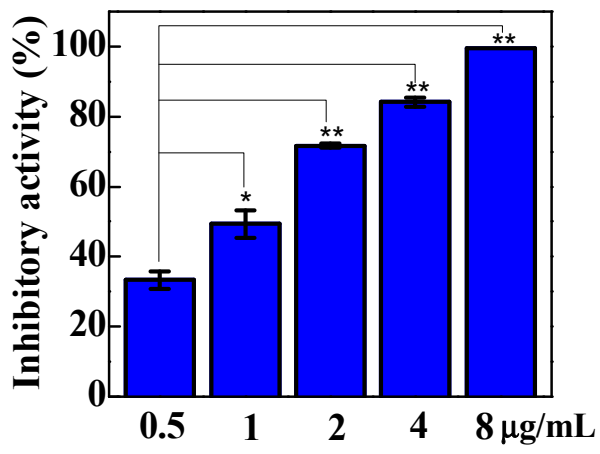

(D)

Figure 4. Images of Escherichia coli (A) and Staphylococcus aureus (C) on TSA plates after $24 \mathrm{~h}$ of incubation after treatment with different concentrations of TC/PG nanocomposites, and the corresponding inhibition activity against E. coli (B) and S. aureus (D).

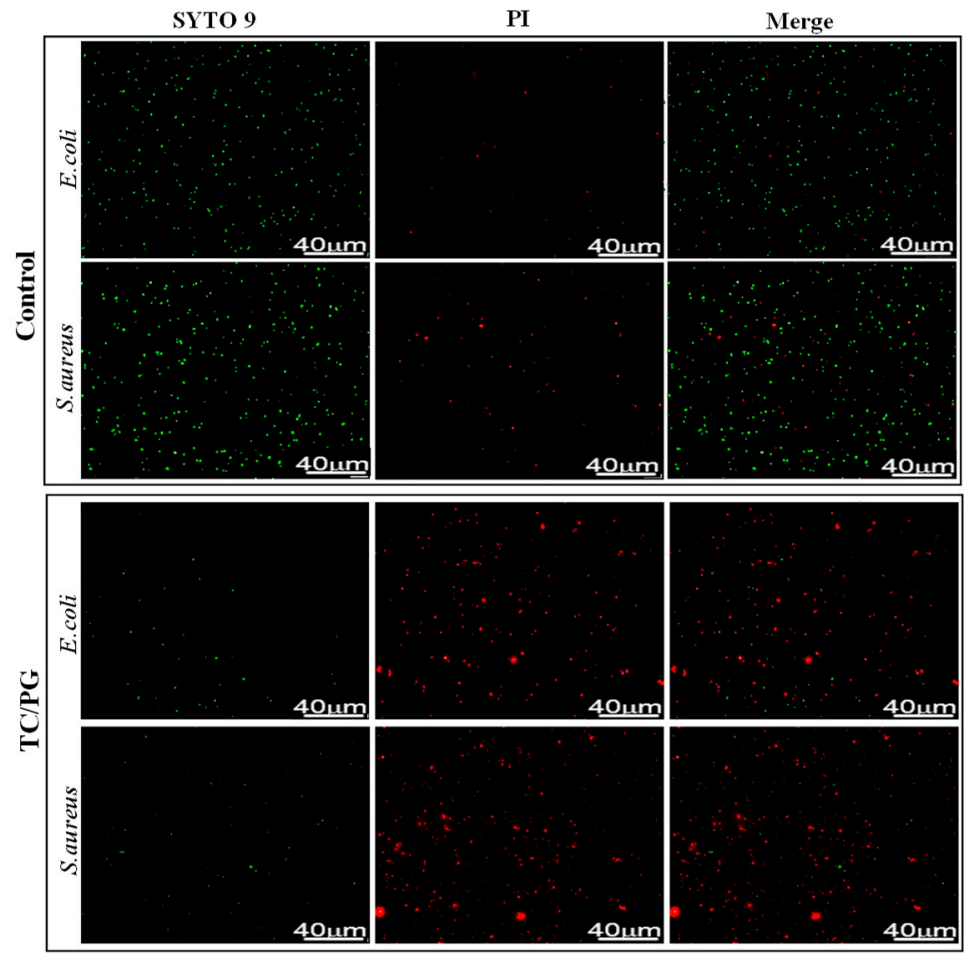

Figure 5. Images of the stained bacteria. 
The antibacterial activity of the fabricated TC/PG nanocomposites was evaluated by the disk diffusion method. The size of the inhibition zone directly reflects the bacterial inhibition ability since the inhibition zone is generated by the inhibition effect of the tested material on bacterial growth [42]. Images of the inhibition zone of the GO and the TC/PG nanocomposites are shown in Supplementary Figure S1. No noticeable inhibition zone can be found around the GO nanosheets, indicating that the GO nanosheets did not exhibit any inhibition through a leaching effect on E. coli and S. aureus. There are clear inhibition zones around the TC/PG nanocomposites, which exhibit a strong inhibition effect on the tested strains. This phenomenon is due to the TC that was loaded onto the nanocomposites, which can leach out from the TC/PG nanocomposites to inhibit the growth of the tested bacteria. The prepared TC/PG nanocomposites have an excellent inhibition effect.

To further examine the antibacterial effect of the TC/PG nanocomposites, the integrity of the cell membrane after being treated with TC/PG nanocomposites was studied by SEM. As illustrated in Figure 6, native E. coli cells have a rod shape with intact and plump cell walls. The E. coli cells had wrinkled and damaged morphologies after incubation with the TC/PG nanocomposites. A similar result was obtained for $S$. aureus. Native $S$. aureus cells exhibit a plump and round shape with a smooth surface morphology. After being cultured with TC/PG nanocomposites, the shapes of $S$. aureus cells became distorted, and the cells exhibited wrinkled and damaged cell membranes with some intracellular inclusion leakage. So, the TC/PG nanocomposites demonstrate great antibacterial performance on E. coli and S. aureus through the destruction of cell structures with the compromise of the integrity of the bacterial cell membrane and the leakage of cytoplasm.

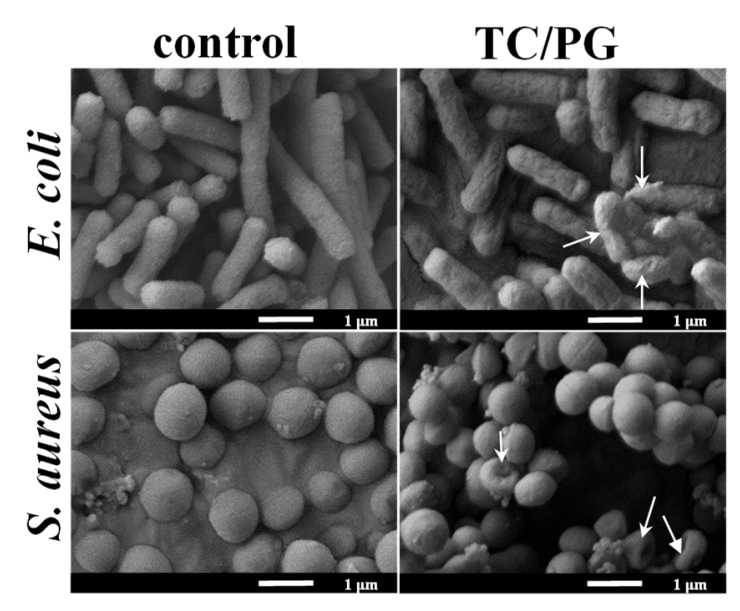

Figure 6. The morphologies after treatment with TC/PG nanocomposites. White arrows point to the damaged cells.

\subsection{Protein Leakage}

The existence of protein in the bacterial suspension represents damage to bacterial cell membranes [43]. The amount of protein leakage was evaluated using a BCA Protein Assay Kit. The BCA protein assay forms a purple-colored and water-soluble BCA/copper complex, which exhibits an absorbance at $562 \mathrm{~nm}$. The result is shown in Figure 7. The detected protein concentration without treatment was $67 \mu \mathrm{g} / \mathrm{mL}$ for E. coli. Some proteins were found to have leaked into the bacterial suspension for the control group, indicating that some membrane damage had occurred. However, a higher protein concentration $(140 \mu \mathrm{g} / \mathrm{mL})$ for the bacterial suspension treated with TC/PG nanocomposites was observed, suggesting that more leakage of cell content had happened. A lower amount of protein leakage, with a concentration of $44 \mu \mathrm{g} / \mathrm{mL}$, was observed for untreated S. aureus. The detected protein concentration in S. aureus with TC/PG nanocomposite treatment was $148 \mu \mathrm{g} / \mathrm{mL}$, which was more than 2 times than that of the control. This phenomenon was due to the enhanced bacterial membrane damage capability of the fabricated TC/PG nanocomposites. Moreover, the TC/PG 
nanocomposites exhibited a greater damaging effect on the cell membranes of S. aureus than E. coli. This is consistent with the results from the disk diffusion experiment.

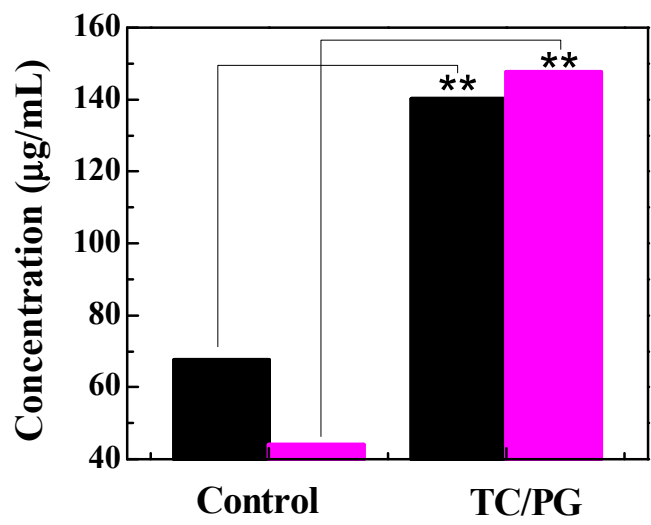

Figure 7. The protein leakage concentration in E. coli (black column) and S. aureus (pink column) suspensions treated with TC/PG nanocomposites.

\subsection{Oxidative Stress-Mediated Antimicrobial Property}

Physical interaction and oxidative stress formation are deemed to be responsible for the antibacterial performance of graphene-based materials [13]. GO nanosheets with sharp edges can puncture or penetrate into the cell membrane by direct contact with bacteria to disrupt their physical integrity, leading to the destruction of the bacterial cell membrane, intracellular component leakage, the uptake of membrane-impermeable dyes, and changes in transmembrane potential [17]. GO nanosheets can induce chemical stress in bacteria in order to generate oxidative stress and degrade the structure of cells regardless of whether any reactive oxygen species (ROS) are produced [44]. The ROS-dependent/independent oxidative stress pathways mediated by TC/PG nanocomposites were determined by the XTT method and an Ellman's assay, respectively. The absorbance at $470 \mathrm{~nm}$ reflects the capacity for ROS production. No significant difference was found in the ROS production capacities among the tested samples and the control at $2 \mathrm{~h}, 4 \mathrm{~h}$, or $6 \mathrm{~h}$ of incubation (Figure 8A). Moreover, only trace amounts of superoxide anions were formed by the TC/PG nanocomposites, since we barely detected any absorbance at $470 \mathrm{~nm}$. In other words, the TC/PG nanocomposites did not show the ability to mediate ROS-dependent oxidative stress against bacteria due to their low production of superoxide anions.

The possibility for ROS-independent oxidative stress formed by TC/PG nanocomposites was evaluated using an Ellman's assay. Elman's reagent can quantitate thiols by forming a mixed disulfide that has a maximum absorption at $410 \mathrm{~nm}$ [45]. Glutathione (GSH) losses after incubation with TC/PG nanocomposites for $2-6 \mathrm{~h}$ are illustrated in Figure $8 \mathrm{~B}$. The negative control did not produce any oxidation of GSH. $\mathrm{H}_{2} \mathrm{O}_{2}$, acting as the positive control, caused a very high fraction of glutathione loss with incubation for $2 \mathrm{~h}, 4 \mathrm{~h}$, and $6 \mathrm{~h}$. The GO nanosheets also showed a great capability for GSH oxidation; the glutathione losses were found to be more than $78 \%$. The TC/PG nanocomposites showed a reduced oxidation capacity of $24.4 \pm 0.9 \%, 25.3 \pm 0.7 \%$, and $27.8 \pm 1.5 \%$ for 2,4 , and $6 \mathrm{~h}$ of incubation, respectively. Thus, the fabricated TC/PG nanocomposites were indirectly shown to possess some GSH oxidation ability, which mediates ROS-independent oxidative stress toward bacteria.

In summary, the great bacterial inhibition behavior is due to the synergistic effect of the PG nanosheets and the TC. First, due to the existence of the high-density amine groups in the TC/PG nanocomposites, they can easily adsorb onto cell membranes with a negative charge via electrostatic interactions. Second, a physical interaction occurs upon direct contact with the bacteria and ROS-independent oxidative stress is generated that produces some oxidation capacity. Third and finally, TCH released from the TC/PG nanocomposites effectively restrains the growth of bacteria by inhibiting the synthesis of bacterial protein. 


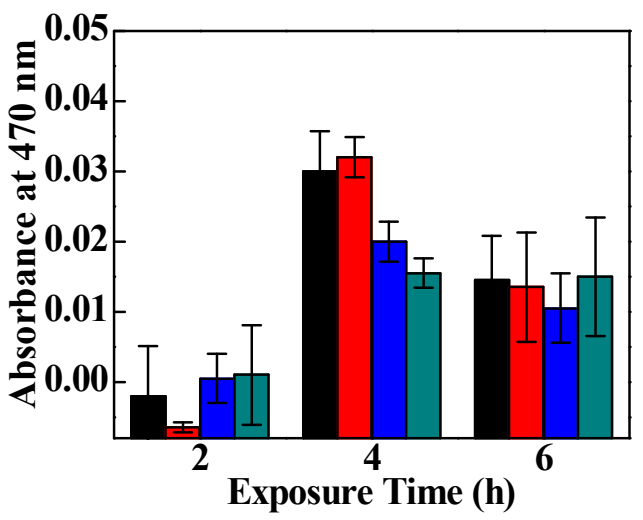

(A)

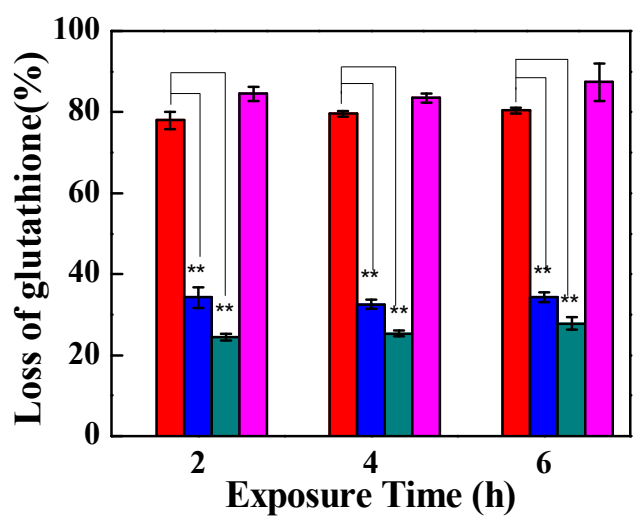

(B)

Figure 8. Oxidative stress mediated by the TC/PG nanocomposites. (A): reactive oxygen species (ROS)-dependent oxidative stress at exposure times of 2-6 h monitored using XTT tests. (B): Loss of glutathione after incubation with TC/PG nanocomposites for 2-6 h. Black columns represent the negative control, red columns represent GO nanosheets, blue columns represent PG nanosheets, and dark cyan columns represent TC/PG nanocomposites. $\mathrm{H}_{2} \mathrm{O}_{2}$ (the magenta column) was used as a positive control.

\section{Conclusions}

In this study, TC-loaded PG nanocomposites were constructed and successfully prepared. The developed TC/PG nanocomposites exhibit a slow and sustained TC release behavior, indicating that PG nanosheets can be utilized as a good TC carrier. Moreover, the TC/PG nanocomposites exhibit a great bacterial inhibition capacity towards $E$. coli and S. aureus. The antibacterial performance relies on the synergistic effect between TC and the PG nanosheets in the nanocomposite. These results clearly demonstrate that the prepared nanocomposites have great potential for application as an antibacterial agent.

Supplementary Materials: The following are available online at http://www.mdpi.com/2079-4991/9/7/913/s1, Figure S1: Inhibition zone pictures of GO, TC/PG and TC against E. coli and S. aureus; Table S1: Diameters $(\mathrm{mm})$ of Inhibition zone of GO, TC/PG and TC against E. coli and S. aureus; Figure S2: XRD spectra of GO and TC/PG nanocomposites.

Author Contributions: Conceptualization, W.S.; methodology, L.J. and Z.Z.; structure analysis, Y.W. and S.Y.; antibacterial investigation, L.J. and C.S.; revision, W.S. and R.Z., writing, W.S.

Funding: This research was funded by Postgraduate Research \&Practice Innovation Program of Jiangsu Province (SJKY19_0871), the 333 Project of Jiangsu Province, the Six Talents Peak Project of Jiangsu Province, and the Priority Academic Program Development of Jiangsu Higher Education Institutions (PAPD). The authors thank the Advanced Analysis and Testing Center of Nanjing Forestry University.

Conflicts of Interest: The authors declare no conflicts of interest.

\section{References}

1. Xie, Q.; Zhang, Y.; Zhao, P. Facile fabrication of honeycomb-like restacking-inhibited graphene architecture with superior electrochemical performance for energy storage. Mater. Lett. 2018, 225, 93-96. [CrossRef]

2. Ye, M.; Gao, J.; Xiao, Y.; Xu, T.; Zhao, Y.; Qu, L. Metal/graphene oxide batteries. Carbon 2017, 125, $299-307$. [CrossRef]

3. Pandit, S.; Cao, Z.; Mokkapati, V.R.; Celauro, E.; Yurgens, A.; Lovmar, M.; Westerlund, F.; Sun, J.; Mijakovic, I. Vertically Aligned Graphene Coating is Bactericidal and Prevents the Formation of Bacterial Biofilms. Adv. Mater. Interfaces 2018, 5, 1701331. [CrossRef]

4. He, D.; Song, L.; Lv, L.; Zhang, Z.; Qu, L. Superelastic air-bubbled graphene foam monoliths as structural buffer for compressible high-capacity anode materials in lithium-ion batteries. Chem. Eng. J. 2018, 331, 704-711. [CrossRef] 
5. Şenol, A.M.; Onganer, Y.; Meral, K. An unusual "off-on" fluorescence sensor for iron(III) detection based on fluorescein-reduced graphene oxide functionalized with polyethyleneimine. Sens. Actuators B Chem. 2017, 239, 343-351. [CrossRef]

6. Nayak, G.S.; Zybala, R.; Kozinski, R.; Woluntarski, M.; Telle, R.; Schickle, K. Immobilization of reduced graphene oxide nano-flakes on inert ceramic surfaces using self-assembled monolayer technique. Mater. Lett. 2018, 225, 109-112. [CrossRef]

7. Qian, W.; Wang, Z.; He, D.; Huang, X.; Su, J. Ornidazole-loaded graphene paper for combined antibacterial materials. J. Saudi Chem. Soc. 2018, 22, 581-587. [CrossRef]

8. Gong, P.; Ji, S.; Wang, J.; Dai, D.; Wang, F.; Tian, M.; Zhang, L.; Guo, F.; Liu, Z. Fluorescence-switchable ultrasmall fluorinated graphene oxide with high near-infrared absorption for controlled and targeted drug delivery. Chem. Eng. J. 2018, 348, 438-446. [CrossRef]

9. Yadav, N.; Kumar, N.; Prasad, P.; Shirbhate, S.; Sehrawat, S.; Lochab, B. Stable Dispersions of Covalently Tethered Polymer Improved Graphene Oxide Nanoconjugates as an Effective Vector for siRNA Delivery. ACS Appl. Mater. Interfaces 2018, 10, 14577-14593. [CrossRef]

10. Barahuie, F.; Saifullah, B.; Dorniani, D.; Fakurazi, S.; Karthivashan, G.; Hussein, M.Z.; Elfghi, F.M. Graphene oxide as a nanocarrier for controlled release and targeted delivery of an anticancer active agent, chlorogenic acid. Mater. Sci. Eng. C 2017, 74, 177-185. [CrossRef]

11. Qiu, J.; Liu, L.; Zhu, H.; Liu, X. Combination types between graphene oxide and substrate affect the antibacterial activity. Bioact. Mater. 2018, 3, 341-346. [CrossRef] [PubMed]

12. Henriques, P.C.; Borges, I.; Pinto, A.M.; Magalhães, F.D.; Gonçalves, I.C. Fabrication and antimicrobial performance of surfaces integrating graphene-based materials. Carbon 2018, 132, 709-732. [CrossRef]

13. Karahan, H.E.; Wang, Y.; Li, W.; Liu, F.; Wang, L.; Sui, X.; Riaz, M.A.; Chen, Y. Antimicrobial graphene materials: The interplay of complex materials characteristics and competing mechanisms. Biomater. Sci. 2018, 6, 766-773. [CrossRef] [PubMed]

14. Zou, X.; Zhang, L.; Wang, Z.; Luo, Y. Mechanisms of the Antimicrobial Activities of Graphene Materials. J. Am. Chem. Soc. 2016, 138, 2064-2077. [CrossRef] [PubMed]

15. Li, Y.F.; Yuan, H.Y.; Von Dem Bussche, A.; Creighton, M.; Hurt, R.H.; Kane, A.B.; Gao, H.J. Graphene microsheets enter cells through spontaneous membrane penetration at edge asperities and corner sites. Proc. Natl. Acad. Sci. USA 2013, 110, 12295-12300. [CrossRef]

16. Liu, S.; Hu, M.; Zeng, T.H.; Wu, R.; Jiang, R.; Wei, J.; Wang, L.; Kong, J.; Chen, Y. Lateral Dimension-Dependent Antibacterial Activity of Graphene Oxide Sheets. Langmuir 2012, 28, 12364-12372. [CrossRef] [PubMed]

17. Perreault, F.; de Faria, A.F.; Nejati, S.; Elimelech, M. Antimicrobial Properties of Graphene Oxide Nanosheets: Why Size Matters. ACS Nano 2015, 9, 7226-7236. [CrossRef]

18. Zhu, J.; Wang, J.; Hou, J.; Zhang, Y.; Liu, J.; Van der Bruggen, B. Graphene-based antimicrobial polymeric membranes: A review. J. Mater. Chem. A 2017, 5, 6776-6793. [CrossRef]

19. Ye, X.L.; Feng, J.; Zhang, J.X.; Yang, X.J.; Liao, X.Y.; Shi, Q.S.; Tan, S.Z. Controlled release and long-term antibacterial activity of reduced graphene oxide/quaternary ammonium salt nanocomposites prepared by non-covalent modification. Colloid Surf. B Biointerfaces 2017, 149, 322-329. [CrossRef]

20. Pan, N.Y.; Liu, Y.; Fan, X.Y.; Jiang, Z.M.; Ren, X.H.; Liang, J. Preparation and characterization of antibacterial graphene oxide functionalized with polymeric N-halamine. J. Mater. Sci. 2017, 52, 1996-2006. [CrossRef]

21. Pang, J.; Kang, Z.; Wang, R.; Xu, B.; Nie, X.; Fan, L.; Zhang, F.; Du, X.; Feng, S.; Sun, D. Exploring the sandwich antibacterial membranes based on UiO-66/graphene oxide for forward osmosis performance. Carbon 2019, 144, 321-332. [CrossRef]

22. Nayamadi Mahmoodabadi, A.; Kompany, A.; Mashreghi, M. Characterization, antibacterial and cytotoxicity studies of graphene- $\mathrm{Fe}_{3} \mathrm{O}_{4}$ nanocomposites and $\mathrm{Fe}_{3} \mathrm{O}_{4}$ nanoparticles synthesized by a facile solvothermal method. Mater. Chem. Phys. 2018, 213, 285-294. [CrossRef]

23. Liang, Y.; Yang, D.S.; Cui, J.H. A graphene oxide/silver nanoparticle composite as a novel agricultural antibacterial agent against Xanthomonas oryzae pv. oryzae for crop disease management. New J. Chem. 2017, 41, 13692-13699. [CrossRef]

24. Zhong, L.; Liu, H.; Samal, M.; Yun, K. Synthesis of ZnO nanoparticles-decorated spindle-shaped graphene oxide for application in synergistic antibacterial activity. J. Photochem. Photobiol. B Biol. 2018, 183, $293-301$. [CrossRef] [PubMed] 
25. Gupta, A.; Holoidovsky, L.; Thamaraiselvan, C.; Thakur, A.K.; Singh, S.P.; Meijler, M.M.; Arnusch, C.J. Silver-doped laser-induced graphene for potent surface antibacterial activity and anti-biofilm action. Chem. Commun. (Camb. Engl.) 2019. [CrossRef] [PubMed]

26. Deng, C.-H.; Gong, J.-L.; Zeng, G.-M.; Zhang, P.; Song, B.; Zhang, X.-G.; Liu, H.-Y.; Huan, S.-Y. Graphene sponge decorated with copper nanoparticles as a novel bactericidal filter for inactivation of Escherichia coli. Chemosphere 2017, 184, 347-357. [CrossRef] [PubMed]

27. Qian, W.H.; Qiu, J.J.; Su, J.S.; Liu, X.Y. Minocycline hydrochloride loaded on titanium by graphene oxide: An excellent antibacterial platform with the synergistic effect of contact-killing and release-killing. Biomater. Sci. 2018, 6, 304-313. [CrossRef] [PubMed]

28. Yang, L.; Jia, F.; Yang, B.; Song, S. Efficient adsorption of $\mathrm{Au}(\mathrm{CN})$ 2- from gold cyanidation with graphene oxide-polyethylenimine hydrogel as adsorbent. Results Phys. 2017, 7, 4089-4095. [CrossRef]

29. Chen, B.A.; Liu, M.; Zhang, L.M.; Huang, J.; Yao, J.L.; Zhang, Z.J. Polyethylenimine-functionalized graphene oxide as an efficient gene delivery vector. J. Mater. Chem. 2011, 21, 7736-7741. [CrossRef]

30. Zhou, X.; Laroche, F.; Lamers, G.E.M.; Torraca, V.; Voskamp, P.; Lu, T.; Chu, F.Q.; Spaink, H.P.; Abrahams, J.P.; Liu, Z.F. Ultra-small graphene oxide functionalized with polyethylenimine (PEI) for very efficient gene delivery in cell and zebrafish embryos. Nano Res. 2012, 5, 703-709. [CrossRef]

31. Wang, N.; Hu, B.; Chen, M.L.; Wang, J.H. Polyethylenimine mediated silver nanoparticle-decorated magnetic graphene as a promising photothermal antibacterial agent. Nanotechnology 2015, 26, 195703. [CrossRef] [PubMed]

32. Jiang, L.; Su, C.; Ye, S.; Wu, J.; Zhu, Z.; Wen, Y.; Zhang, R.; Shao, W. Synergistic antibacterial effect of tetracycline hydrochloride loaded functionalized graphene oxide nanostructures. Nanotechnology 2018, 29, 505102. [CrossRef] [PubMed]

33. Ellman, G.L. Tissue Sulfhydryl Groups. Arch. Biochem. Biophys. 1959, 82, 70-77. [CrossRef]

34. Shao, W.; Liu, X.; Min, H.; Dong, G.; Feng, Q.; Zuo, S. Preparation, characterization, and antibacterial activity of silver nanoparticle-decorated graphene oxide nanocomposite. ACS Appl. Mater. Interfaces 2015, 7, 6966-6973. [CrossRef] [PubMed]

35. Tang, J.; Hong, J.; Liu, Y.; Wang, B.; Hua, Q.; Liu, L.; Ying, D. Urea Controlled-Release Fertilizer Based on Gelatin Microspheres. J. Polym. Environ. 2017, 26, 1930-1939. [CrossRef]

36. Liu, K.; Lin, X.; Chen, L.; Huang, L.; Cao, S.; Wang, H. Preparation of microfibrillated cellulose/chitosan-benzalkonium chloride biocomposite for enhancing antibacterium and strength of sodium alginate films. J. Agric. Food Chem. 2013, 61, 6562-6567. [CrossRef] [PubMed]

37. Vo, D.-T.; Whiteley, C.G.; Lee, C.-K. Hydrophobically Modified Chitosan-Grafted Magnetic Nanoparticles for Bacteria Removal. Ind. Eng. Chem. Res. 2015, 54, 9270-9277. [CrossRef]

38. Bilbao-Sainz, C.; Chiou, B.-S.; Valenzuela-Medina, D.; Du, W.-X.; Gregorski, K.S.; Williams, T.G.; Wood, D.F.; Glenn, G.M.; Orts, W.J. Solution blow spun poly(lactic acid)/hydroxypropyl methylcellulose nanofibers with antimicrobial properties. Eur. Polym. J. 2014, 54, 1-10. [CrossRef]

39. Zhao, R.; Kong, W.; Sun, M.; Yang, Y.; Liu, W.; Lv, M.; Song, S.; Wang, L.; Song, H.; Hao, R. Highly Stable Graphene-Based Nanocomposite (GO-PEI-Ag) with Broad-Spectrum, Long-Term Antimicrobial Activity and Antibiofilm Effects. ACS Appl. Mater. Interfaces 2018, 10, 17617-17629. [CrossRef]

40. Liu, Y.; Wang, L.; Yang, L.; Zhan, Y.; Zou, L.; Ye, B. Nonenzymatic $\mathrm{H}_{2} \mathrm{O}_{2}$ Electrochemical Sensor Based on SnO2-NPs Coated Polyethylenimine Functionalized Graphene. Electroanalysis 2017, 29, 2044-2052. [CrossRef]

41. Shao, W.; Liu, H.; Wang, S.; Wu, J.; Huang, M.; Min, H.; Liu, X. Controlled release and antibacterial activity of tetracycline hydrochloride-loaded bacterial cellulose composite membranes. Carbohydr. Polym. 2016, 145, 114-120. [CrossRef]

42. Li, G.W.; Yu, S.M.; Xue, W.; Ma, D.; Zhang, W. Chitosan-graft-PAMAM loading nitric oxide for efficient antibacterial application. Chem. Eng. J. 2018, 347, 923-931. [CrossRef]

43. Zhao, R.; Lv, M.; Li, Y.; Sun, M.; Kong, W.; Wang, L.; Song, S.; Fan, C.; Jia, L.; Qiu, S.; et al. Stable Nanocomposite Based on PEGylated and Silver Nanoparticles Loaded Graphene Oxide for Long-Term Antibacterial Activity. ACS Appl. Mater. Interfaces 2017, 9, 15328-15341. [CrossRef] [PubMed] 
44. Kim, T.I.; Kwon, B.; Yoon, J.; Park, I.J.; Bang, G.S.; Park, Y.; Seo, Y.S.; Choi, S.Y. Antibacterial Activities of Graphene Oxide-Molybdenum Disulfide Nanocomposite Films. ACS Appl. Mater. Interfaces 2017, 9, 7908-7917. [CrossRef] [PubMed]

45. Liu, S.; Zeng, T.H.; Hofmann, M.; Burcombe, E.; Wei, J.; Jiang, R.; Kong, J.; Chen, Y. Antibacterial Activity of Graphite, Graphite Oxide, Graphene Oxide, and Reduced Graphene Oxide: Membrane and Oxidative Stress. ACS Nano 2011, 5, 6971-6980. [CrossRef] [PubMed] 